\title{
BMJ Open Pramipexole in peritoneal dialysis patients with restless legs syndrome (RLS): a protocol for a multicentre double-blind randomised controlled trial
}

$\overline{\text { Tian-tian Ma (D) , }{ }^{1} \text { Zhikai Yang, }{ }^{2} \text { Sainan Zhu, }{ }^{3} \text { Jing-hong Zhao, }{ }^{4} \mathrm{Yi} \mathrm{Li}^{5} \text { Fu-yun Sun, }{ }^{6}}$ Nan Zhao, ${ }^{6}$ Zu-ying Xiong, ${ }^{7}$ Zi-bo Xiong, ${ }^{7}$ Jie Dong $^{8}$

To cite: Ma T, Yang Z, Zhu S, et al. Pramipexole in peritoneal dialysis patients with restless legs syndrome (RLS): a protocol for a multicentre double-blind randomised controlled trial. BMJ Open 2020;10:e033815. doi:10.1136/ bmjopen-2019-033815

- Prepublication history for this paper is available online. To view these files, please visit the journal online (http://dx.doi. org/10.1136/bmjopen-2019033815).

Received 25 August 2019 Revised 22 January 2020 Accepted 29 January 2020
Check for updates

(C) Author(s) (or their employer(s)) 2020. Re-use permitted under CC BY-NC. No commercial re-use. See rights and permissions. Published by BMJ.

For numbered affiliations see end of article.

Correspondence to

Dr Jie Dong;

jie.dong@bjmu.edu.cn

\section{ABSTRACT}

Introduction Restless legs syndrome (RLS) is a common neurological sensorimotor disorder among patients with end stage renal disease. This clinical trial aimed to provide evidence on the efficacy and safety of pramipexole in patients with uremic RLS receiving peritoneal dialysis (PD). Methods and analysis This is a 12-week, multicentre, randomised, double-blind, placebo-controlled clinical trial. In total, 104 patients with uremic RLS receiving PD will be enrolled from four hospitals and randomly assigned in a 1:1 ratio to either placebo or pramipexole. We will determine the efficacy of pramipexole in the improvement of International RLS Study Group Rating Scale as the primary outcome, while responder rates for other RLS scales at week 12, change from baseline to week 12 for psychological status, sleep disorder and quality of life and blood pressure represent the secondary outcomes.

Ethics and dissemination The study was approved by the ethics committees of Peking University First Hospital, Xinqiao hospital of Army Medical University, Cangzhou Center Hospital and Peking University Shenzhen Hospital. The results will be disseminated in peer-reviewed journals. Trial registration number NCT03817554

\section{INTRODUCTION}

Restless legs syndrome (RLS) is a common neurological sensorimotor disorder, characterised by an uncontrollable urge to move one's limbs, with symptoms predominant at night or at periods of rest. According to the diagnostic criteria for RLS proposed by the International RLS Study Group (IRLSSG), the diagnosis is mainly based on patients subjective symptoms and feelings, and an RLS severity rating scale is used to evaluate the severity of symptoms. ${ }^{1}$

The morbidity of RLS among patients with end stage renal disease (ESRD) was approximately $30 \%$ (range $7 \%-45 \%$ ), ${ }^{2}$ and there was no difference regarding the frequency of RLS between patients on hemodialysis
Strengths and limitations of this study

- This is the first double-blinded randomised controlled trial to explore whether pramipexole could improve the symptoms of restless legs syndrome (RLS) in patients receiving peritoneal dialysis (PD).

- The design for patient enrollment and follow-up is rigorous and feasible. The double-blinded assignment of intervention in this trial is also applicable.

- The findings from this study will provide robust evidence of the efficacy and safety of pramipexole to treat RLS in patients receiving PD.

- We are aware of the disadvantages of evaluating RLS using subjective questionnaires as the primary outcome.

(HD) and those receiving peritoneal dialysis (PD). ${ }^{3}{ }^{4}$ Compared with those without RLS, dialysis patients with RLS had impaired quality of life and higher short-term mortality. ${ }^{56}$ The precise mechanisms of uremic RLS are not completely understood. Dysfunction of the dopaminergic system, reduced iron stores in specific regions of the brain, calcium/ phosphate imbalance and subclinical peripheral nerve abnormalities are possible factors implicated in the pathophysiology of uremic RLS. ${ }^{2-11}$ The management of RLS includes pharmacological and non-pharmacological therapies. The first-line treatment includes non-ergot dopamine agonist, that is, pramipexole and rotigotine, and $\alpha-2-\delta$ calcium channel ligands, such as gabapentin. ${ }^{2}{ }^{12}$ For patients with severe RLS symptoms, opioids may be prescribed as monotherapy or as add-on treatment. ${ }^{14}$

Pramipexole, a non-ergoline dopamine agonist with selective affinity for the D2/D3 receptor, could reduce dopamine release and consequently improve both motor and 
non-motor syndromes. ${ }^{15}$ A meta-analysis involving 12 randomised controlled trials (RCTs) including 3286 participants with primary moderate-to-severe primary RLS indicated that pramipexole could effectively improve the symptoms of RLS and sleep quality. ${ }^{16}$ Furthermore, pramipexole also contributed to improvements in RLSrelated mood disturbance. ${ }^{17}$ For patients with uremic RLS receiving HD, although pramipexole and ropinirole showed some benefits on ameliorating the symptoms of RLS in single-centre studies, ${ }^{18} 19$ a review of 220 patients receiving HD did not determine the effects of nonergot dopamine agonist on RLS. ${ }^{20}$ There have been no studies in terms of pharmacological interventions of RLS performed in patients receiving PD.

Therefore, the current trial is designed to evaluate the efficacy and safety of pramipexole in patients with uremic RLS receiving PD via a multicentre, randomised, doubleblind and placebo-controlled clinical trial.

\section{MATERIALS AND METHODS}

Trial design and setting

This is a 12-week, multicentre, randomised, double-blind, placebo-controlled clinical trial. In total, 104 patients with uremic RLS receiving PD from Peking University First Hospital, Xinqiao hospital of Army Medical University, Cangzhou Center Hospital and Peking University Shenzhen Hospital will be enrolled and randomly assigned in a 1:1 ratio to receive either placebo or pramipexole.

The flow chart of the study is presented in figure 1 .

\section{Study population}

Inclusion criteria include:

- Diagnosed with RLS according to the IRLSSG criteria. ${ }^{5}$

- Moderate to severe symptoms, as indicated by a baseline score on the IRLSSG Rating Scale (IRLS) of $>15$.

- Chronic PD for at least 3 months.

- Age $\geq 18$ years.

- With written informed consent to participate in the study.

Exclusion criteria include:

- Severe gastrointestinal tract disease in which the patient cannot tolerate the treatment.

- Difficulty in undergoing any procedure listed in the protocol.

- Taking medications (eg, dopaminergic receptor agonists/antagonists, tricyclic antidepressants, lithium) that might possibly influence RLS

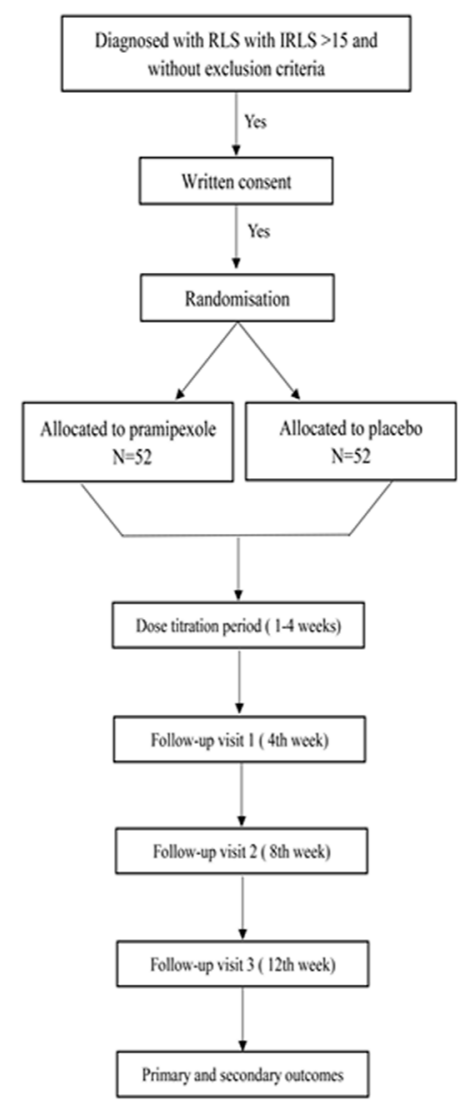

Figure 1 Flow chart of the participants in the study. IRLS, International RLS Study Group Rating Scale; RLS, restless legs syndrome. 
symptoms, sleep disorder (eg, hypersomnolence, severe insomnia, circadian rhythm disorders, sleepdisordered breathing, parasomnias) or mood disturbance within 14 days.

- Iron deficiency (ferritin $<100 \mathrm{ng} / \mathrm{mL})$, anaemia $(\mathrm{Hb}<90 \mathrm{~g} / \mathrm{L})$.

- Inadequate dialysis $(\mathrm{Kt} / \mathrm{V}<1.7$ per week).

- Acute comorbidities within 1 month, including acute infection, acute cardiovascular and cerebrovascular diseases, active hepatitis and connective tissue diseases.

- Radiation and chemotherapy for cancer within 1 year.

- Pregnant or breastfeeding women.

- Drug or alcohol abuse within 1 year.

- Patients with a psychiatric history.

\section{Randomisation and blinding}

An external statistical consultant will undertake the computer-generated randomisation for the order of the intervention in blocks of 4 , which will be blinded to the investigators. Eligible patients will be randomised to one of the two groups in a 1:1 ratio. To ensure all investigators and participants are blinded to the treatment, study medications will be packaged off-site by an external pharmacy with a generic label. The data will be unalterable, allowing the randomisation process to be audited. All laboratory parameters and evaluations on questionnaires for all participants will be measured by staff who are also blinded to the treatment assignment.

\section{Experimental intervention}

Pramipexole or placebo will be taken once daily in the evening, 2-3 hours before bedtime. The starting dose of pramipexole or placebo is $0.125 \mathrm{mg} /$ day. During the first 4 weeks, the daily dose could be increased by the treating physician in weekly intervals to $0.25,0.50$ or $0.75 \mathrm{mg}$ /day, according to the Patient Global Impression (PGI) Scale rating and the overall tolerability of the drug. In the case of adverse events (AEs), the dose could be reduced to the previous dose step. During 5-12 weeks, the dose will remain constant.

\section{Control}

Participants in the control group will receive a matched placebo. During the first 4 weeks, the daily dose could be increased by the treating physician in weekly intervals to $0.25,0.50$ or $0.75 \mathrm{mg} /$ day, according to the PGI Scale rating and the overall tolerability of the drug. In the case of AEs, the dose could be reduced to the previous dose step. During 5-12 weeks, the dose will remain constant.

\section{Concomitant treatment}

For both the intervention and control groups, all dialysis and other medication prescriptions will be in accordance with routine clinical care and International Society for Peritoneal Dialysis guideline recommendations.

\section{Baseline evaluation and follow-up}

Data collection for this study comprises two phases: baseline evaluation and follow-up. All the data will be recorded by the responsible physician in a uniform case report form (CRF). Detailed information collected in each phase is described below.

\section{Baseline evaluation}

For all patients, information including demographic data (age, gender, body mass index, education, career, income and Charlson Score), dialysis duration, primary disease, comorbidities, current medication, laboratory parameters, IRLS, Clinician Global Impression (CGI)-I Scale, PGI Scale, Self-rating Anxiety Scale (SAS) Score, Selfrating Depression Scale (SDS) Score, Medical Outcomes Study (MOS) Sleep Scale, Epworth Sleepiness Scale (ESS) Score, Quality of Life (QOL) (KDQOLTM-36) Score and 24 hours ambulatory blood pressure will be evaluated.

\section{Follow-up and outcome measures}

On week 12, laboratory parameters, IRLS, CGI-I Scale, PGI Scale, SAS Score, SDS Score, MOS Sleep Scale, ESS Score, QOL (KDQOLTM-36) Score and 24 hours ambulatory blood pressure will be evaluated again.

The primary outcome measure will be the change from baseline to week 12 in the IRLS.

Secondary outcome measures include the following:

- Responder rate (per cent of patients with at least a $50 \%$ reduction in score) for IRLS at week 12.

- Responder rates (per cent at least 'much improved') for CGI-I and PGI Scales at week 12.

- Change from baseline to week 12 for the SAS and SDS Scores.

- Change from baseline to week 12 for three additional MOS Sleep Scale dimensions.

- Change from baseline to week 12 for the ESS Score.

- Change from baseline to week 12 for the QOL (KDQOLTM-36) Score.

- Change from baseline to week 12 for the 24 hours ambulatory blood pressure value, including mean blood pressure in the daytime and nighttime, maximum systolic blood pressure in the daytime and nighttime and maximum diastolic blood pressure in the daytime and night time.

To determine the mean post-treatment change from the baseline, all of the increased data will be presented as positive numbers, and all of the decreased data will be presented as negative numbers.

\section{Safety monitoring}

AEs will be recorded prospectively for all participants. AEs will be recorded in terms of potential causality and severity. AEs will be recorded on the CRF. Severe AEs will be formally reported to the sponsor and principal investigator so that reasonable action can be taken. The potential cause of the AEs in relation to the experimental drug will be assessed and classified into six degrees (unrelated, 
unlikely to be related, possibly related, most likely related, definitely related and unable to assess).

\section{Biochemical testing methods}

In all PD centres, blood samples will be routinely analysed at each visit. Biochemical profiles, including haemoglobin, serum level of calcium, phosphorus, intact parathyroid hormone and ferritin, will be tested using a Hitachi automatic chemistry analyzer (Hitachi, Tokyo, Japan).

\section{Patient and public involvement}

Patients and the public were not involved in the design of the study.

\section{Sample size calculation}

Sample size was planned so that the hypothesised superiority of pramipexole over placebo could be detected at a $2.5 \%$ significance level with $80 \%$ power for the primary outcome. Based on data from a meta-analysis, the change from baseline to post-treatment on the IRLS Score was significantly superior to that of the placebo group (weighted mean difference) $=-4.64 ; 95 \% \mathrm{CI},-5.95$ to $-3.33 ; n=8)$. More patients in the pramipexole group reported at least a $50 \%$ reduction in the IRLS Score after treatment (risk ratio) $=1.57 ; 95 \% \mathrm{CI}, 1.43$ to $1.73 ; \mathrm{n}=8) .{ }^{14}$ This implied that 86 patients in total were required for randomisation to the two treatment groups. Assuming a screening failure rate and drop-out rate of as much as $10 \%$, respectively, it was estimated that up to 104 patients should be recruited.

\section{Statistical analyses}

Data will be expressed as the mean \pm SD (for data that were normally distributed) or the median and IQR (for data that were not normally distributed). A general linear model will be built to examine the trend of change in primary and secondary outcome measures between the pramipexole and control groups, from baseline to the end of the 12 weeks of intervention. The relationship between the dose of pramipexole or placebo and the examined variables will be assessed using Pearson or Spearman rank correlation tests. Data will be censored at the time of study completion, permanent transfer to HD, renal transplantation, spontaneous recovery of dialysis-independent renal function or loss to follow-up.

Statistical analyses will be performed using the SPSS V.20.0. All probabilities will be two-tailed, and the level of significance will be set at 0.05 to reject the null hypothesis.

\section{Ethics and dissemination}

The results will be disseminated in peer-reviewed journals.

\section{DISCUSSION}

RLS, a common and severe complication, is verified to interfere with the daily lives of patients with uremia and is associated with worse outcome in this population. ${ }^{56}$ For pramipexole, a first-line drug for moderate-to-severe RLS in the general population, ${ }^{212}$ it is unclear whether it can be prescribed in patients receiving PD. Only one nonRCT performed in nine patients receiving HD indicated remarkable improvements in the severity of RLS and periodic leg movements, as evaluated using polysomography. ${ }^{18}$ Thus, the present multicentre double-blind RCT is designed to provide evidence on the efficacy and safety of pramipexole in patients with uremic RLS receiving PD. To the best of our knowledge, this study is the first RCT to determine the role of pramipexole in patients with uremic RLS receiving PD. With the prescription of pramipexole in patients with uremic RLS receiving PD over a 12-week study period, we will determine the efficacy of pramipexole to improve IRLS as primary outcome, while responder rates for other RLS scales at week 12, change from baseline to week 12 for psychological status, sleep disorder, quality of life and blood pressure represent the secondary outcomes.

Clinically, $90 \%$ of pramipexole is excreted renally as an unchanged drug $^{21}$; therefore, it is recommended to reduce the daily dose in patients on dialysis with a longer titration interval (ie, 14 days). However, the suitable initial dose and maintenance dose of pramipexole, and the minimum interval to assess an effect before increasing the dose in patients on dialysis has not been determined. As previously reported in patients receiving $\mathrm{HD},{ }^{18} 0.125 \mathrm{mg} /$ day as a starting dose, with optional upward titration according to response and tolerance to a maximum daily dose of $0.75 \mathrm{mg}$, did not indicate severe side effects. Therefore, we will apply the same dosing regime of pramipexole with 4-week intervals for titration in the present PD study. Regarding the treatment duration, among 12 trials on the efficacy of pramipexole in the general population, participants were treated for 3 weeks in one trial, 6-8 weeks in five trials, 12 weeks in five trials and 26 weeks in one trial, yielding a mean treatment duration of 11.12 weeks/person. ${ }^{16}$ The evidence for the treatment duration for patients on dialysis is limited; therefore, we decided to observe a 12-week study period for patients on PD, based on the mean treatment duration in the general population.

In the present study, we will use the post-treatment changes in the IRLS Score to evaluate symptom changes, and responder rates for the IRLS, CGI-I and PGI Scores to determine the extent of improvements. According to the described end points in a systemic review, most trials have chosen similar outcome measurements to evaluate the improvement in RLS. ${ }^{16}$ Polysomography could be applied to evaluate periodic leg movements; however, only a few studies have used it as an outcome measurement. ${ }^{1822}$ The limitations of polysomography in terms of applicability and portability restrict its use among most trials performed out of hospitals, especially when studies need to recruit large samples to achieve adequate statistical power.

All study investigators and staff members will undergo a training programme to teach them the methods and processes of the study. In each centre, there will be an 
observer who will receive unified and standardised training to assess the presence of RLS in patients receiving PD. Consistency checking will be employed among all observers to confirm the uniformity of assessment.

RLS is a common comorbidity that lacks effective treatment among patients with ESRD; therefore, there is an urgent need to explore new strategies. Our study is novel in its assessment of the effect of pramipexole for patients with uremic RLS receiving PD, which will provide clinicians with evidence for the choice of pharmacological therapy of uremic RLS. Despite the experimental nature of the dosing regime and treatment duration of pramipexole applied in this study, the findings of this study will provide clues to further explore suitable therapeutic strategies to treat uremic RLS.

\section{Author affiliations}

${ }^{1}$ Renal Division, Department of Medicine, Peking University First Hospital, Beijing, China

${ }^{2}$ Nephrology, Peking University First Hospital, Beijing, China

${ }^{3}$ Department of Statistics, Peking University First Hospital, Beijing, China

${ }^{4}$ Renal Division, Department of Medicine, Xinqiao Hospital, Chongqing, Sichuan, China

${ }^{5}$ Renal Division, Department of Medicine, Xinqiao Hospital, Chongqing, Sichuan,

China

${ }^{6}$ Renal Division, Department of Medicine, Cang Zhou Central Hospital, Cang Zhou, China

${ }^{7}$ Renal Division, Department of Medicine, Peking University Shenzhen Hospital, Shenzhen, Guangdong, China

${ }^{8}$ Peking University First Hospital, Beijing, China

Acknowledgements The authors would like to express their appreciation to Rong Chu (Civil Aviation General Hospital), Yuan Chen (Peritoneal Dialysis Centers of Peking University First Hospital) and Di Song (Peritoneal Dialysis Centers of Peking University First Hospital).

Contributors T-TM, Z-KY and JD conceived and designed the study. S-NZ calculated the sample size and performed the randomisation. T-TM, Z-KY, JD, J-HZ, YL, F-YS, NZ, Z-YX and Z-BX were responsible for developing the intervention. TM and JD wrote the manuscript. All authors reviewed the protocol as well as this manuscript.

Funding The authors have not declared a specific grant for this research from any funding agency in the public, commercial or not-for-profit sectors.

Competing interests None declared.

Patient consent for publication Not required.

Ethics approval The research will be performed in compliance with the Declaration of Helsinki and ethical approval has been obtained through the ethics committees of Peking University First Hospital, Xinqiao hospital of Army Medical University, Cangzhou Center Hospital and Peking University Shenzhen Hospital.

Provenance and peer review Not commissioned; externally peer reviewed.

Open access This is an open access article distributed in accordance with the Creative Commons Attribution Non Commercial (CC BY-NC 4.0) license, which permits others to distribute, remix, adapt, build upon this work non-commercially, and license their derivative works on different terms, provided the original work is properly cited, appropriate credit is given, any changes made indicated, and the use is non-commercial. See: http://creativecommons.org/licenses/by-nc/4.0/.

ORCID iD

Tian-tian Ma http://orcid.org/0000-0002-5403-1000
REFERENCES

1 Allen RP, Picchietti D, Hening WA, et al. Restless legs syndrome: diagnostic criteria, special considerations, and epidemiology. A report from the restless legs syndrome diagnosis and epidemiology workshop at the National Institutes of health. Sleep Med 2003:4:101-19.

2 Giannaki CD, Hadjigeorgiou GM, Karatzaferi C, et al. Epidemiology, impact, and treatment options of restless legs syndrome in endstage renal disease patients: an evidence-based review. Kidney Int 2014;85:1275-82.

3 Unruh ML, Levey AS, D'Ambrosio C, et al. Restless legs symptoms among incident dialysis patients: association with lower quality of life and shorter survival. Am J Kidney Dis 2004;43:900-9.

4 Losso RLM, Minhoto GR, Riella MC. Sleep disorders in patients with end-stage renal disease undergoing dialysis: comparison between hemodialysis, continuous ambulatory peritoneal dialysis and automated peritoneal dialysis. Int Urol Nephrol 2015;47:369-75.

5 Tekdöș Demircioğlu D, Kavadar G, Esen Öre Özgül, et al. Relationship between restless leg syndrome and quality of life in uremic patients. Agri 2015;27:73-8.

6 La Manna G, Pizza F, Persici E, et al. Restless legs syndrome enhances cardiovascular risk and mortality in patients with endstage kidney disease undergoing long-term haemodialysis treatment. Nephrol Dial Transplant 2011;26:1976-83.

7 Zucconi M, Manconi M, Ferini Strambi L. Aetiopathogenesis of restless legs syndrome. Neurol Sci 2007;28:S47-52.

8 Allen RP. Controversies and challenges in defining the etiology and pathophysiology of restless legs syndrome. Am J Med 2007;120:S13-21.

9 Takaki J, Nishi T, Nangaku M, et al. Clinical and psychological aspects of restless legs syndrome in uremic patients on hemodialysis. Am J Kidney Dis 2003;41:833-9.

10 Collado-Seidel V, Kohnen R, Samtleben W, et al. Clinical and biochemical findings in uremic patients with and without restless legs syndrome. Am J Kidney Dis 1998;31:324-8.

11 Marconi S, Scaglione C, Pizza F, et al. Group I nonreciprocal inhibition in restless legs syndrome secondary to chronic renal failure. Parkinsonism Relat Disord 2012;18:362-6.

12 Silber MH, Becker PM, Earley C, et al. Willis-Ekbom disease Foundation revised consensus statement on the management of restless legs syndrome. Mayo Clin Proc 2013;88:977-86.

13 de Biase S, Pellitteri G, Gigli GL, et al. Advancing synthetic therapies for the treatment of restless legs syndrome. Expert Opin Pharmacother 2019;20:1971-80.

14 de Biase S, Merlino G, Valente M, et al. Opioids in the treatment of restless legs syndrome: pharmacological and clinical aspects. Expert Opin Drug Metab Toxicol 2016;12:1035-45.

15 Antonini A, Calandrella D. Pharmacokinetic evaluation of pramipexole. Expert Opin Drug Metab Toxicol 2011;7:1307-14.

16 Liu GJ, Wu L, Lin Wang S, et al. Efficacy of pramipexole for the treatment of primary restless leg syndrome: a systematic review and meta-analysis of randomized clinical trials. Clin Ther 2016;38:162-79.

17 Montagna P, Hornyak M, Ulfberg J, et al. Randomized trial of pramipexole for patients with restless legs syndrome (RLS) and RLSrelated impairment of mood. Sleep Med 2011;12:34-40.

18 Miranda M, Kagi M, Fabres L, et al. Pramipexole for the treatment of uremic restless legs in patients undergoing hemodialysis. Neurology 2004;62:831-2

19 Pellecchia MT, Vitale C, Sabatini M, et al. Ropinirole as a treatment of restless legs syndrome in patients on chronic hemodialysis: an open randomized crossover trial versus levodopa sustained release. Clin Neuropharmacol 2004;27:178-81.

20 Gopaluni S, Sherif M, Ahmadouk NA. Interventions for chronic kidney disease-associated restless legs syndrome. Cochrane Database Syst Rev 2016;11:Cd010690.

21 Wright CE, Sisson TL, Ichhpurani AK, et al. Steady-state pharmacokinetic properties of pramipexole in healthy volunteers. $J$ Clin Pharmacol 1997;37:520-5.

22 Montplaisir J, Nicolas A, Denesle R, et al. Restless legs syndrome improved by pramipexole: a double-blind randomized trial. Neurology 1999;52:938-43. 